\title{
A new polyketide, penicillolide from the marine-derived fungus Penicillium
}

\section{sacculum}

Tao Liu ${ }^{a}{ }^{a}$, Songya Zhang ${ }^{b}$, Zhanlin Li $^{b}$, Yu Wang ${ }^{a}$, Zaixing Chen ${ }^{c}$, Jiao Bai ${ }^{b}$, Li

Tian $^{\text {de }}$, Yuehu Pei ${ }^{\mathrm{b}}$ and Huiming Hua ${ }^{\mathrm{b}}$

${ }^{a}$ Department of Natural Products Chemistry, School of Pharmacy, China Medical University, Shenyang 110122,

China $;{ }^{b}$ Key Laboratory of Structure-Based Drug Design \& Discovery, Ministry of Education, School of Traditional

Chinese Materia Medica, Shenyang Pharmaceutical University, Shenyang 110016, China; ${ }^{c}$ Department of the

Central Laboratory, School of Pharmacy, China Medical University, Shenyang 110122, China; ${ }^{d}$ The First Institute

of Oceanography SOA, Qingdao 266061, China; ${ }^{e}$ Department of Biology, Qingdao University of Science \&

Technology, Qingdao 266042, China

*taoliu0307@163.com

A new polyketide, penicillolide (1) was isolated from the fermentation broth of the marine-derived fungus Penicillium sacculum GT-308. Compound $\mathbf{1}$ is a polyketide with a unique carbon skeleton. The structure of this compound was established via extensive spectroscopic analyses including 1D-, 2D-NMR, and HRESI-MS.

Keywords: marine-derived fungus; Penicillium sacculum; polyketide; penicillolide 


\section{List}

Figure S1. The ${ }^{1} \mathrm{H}-\mathrm{NMR}$ Spectrum of Compound $\mathbf{1}$ in $\mathrm{CDCl}_{3}$.

Figure S2. The ${ }^{13} \mathrm{C}-\mathrm{NMR}$ Spectrum of Compound $\mathbf{1}$ in $\mathrm{CDCl}_{3}$.

Figure S3. The HSQC Spectrum of Compound 1 in $\mathrm{CDCl}_{3}$.

Figure S4. The HMBC Spectrum of Compound 1 in $\mathrm{CDCl}_{3}$.

Figure S5. The ${ }^{1} \mathrm{H}-{ }^{1} \mathrm{H}$ COSY Spectrum of Compound 1 in $\mathrm{CDCl}_{3}$.

Figure S6. The NOESY Spectrum of Compound 1 in $\mathrm{CDCl}_{3}$.

Figure S7. The ESI-MS Spectrum of Compound 1.

Figure S8. The HRESI-MS Spectrum of Compound $\mathbf{1}$.

Figure S9. Key HMBC and ${ }^{1} \mathrm{H}-{ }^{1} \mathrm{H}$ COSY correlations of compound $\mathbf{1 .}$

Figure S10. Key NOESY correlations of compound 1.

Table S1. NMR spectroscopic data of compound 1 (in $\mathrm{CDCl}_{3}$ ) 
Figure S1. The ${ }^{1} \mathrm{H}-\mathrm{NMR}$ Spectrum of Compound $\mathbf{1}$ in $\mathrm{CDCl}_{3}$.

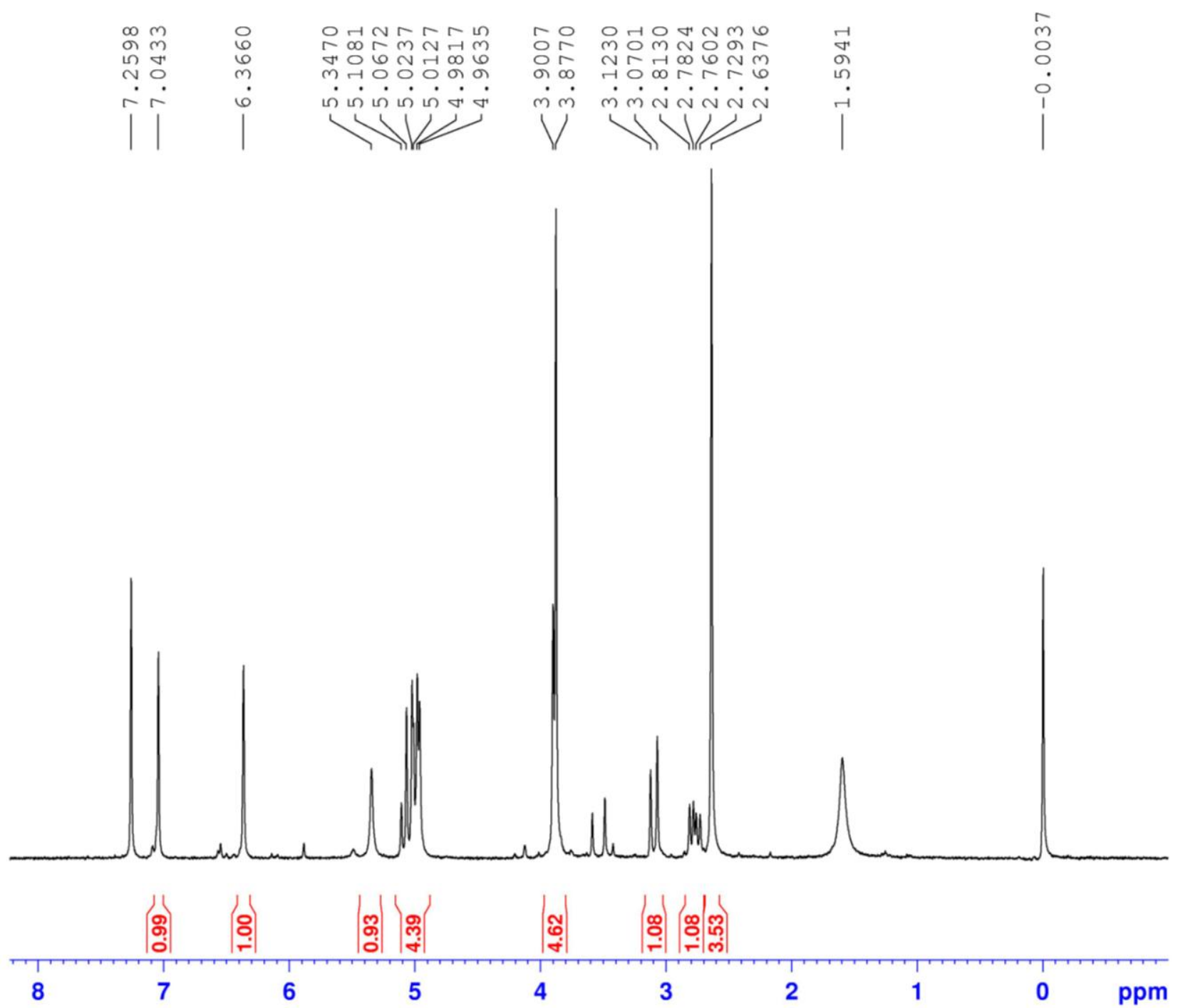


Figure S2. The ${ }^{13} \mathrm{C}-\mathrm{NMR}$ Spectrum of Compound $\mathbf{1}$ in $\mathrm{CDCl}_{3}$.

\begin{tabular}{|c|c|c|c|c|c|}
\hline $\begin{array}{l}\mathscr{2} \\
\infty \\
\infty \\
\infty\end{array}$ & $\begin{array}{l}2 \\
\sigma \\
\infty \\
\infty \\
1\end{array}$ & $\frac{5}{8}$ & $\begin{array}{l}\infty \\
\alpha \\
\stackrel{\sigma}{+} \\
\end{array}$ & 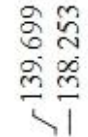 & 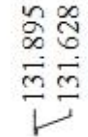 \\
\hline
\end{tabular}

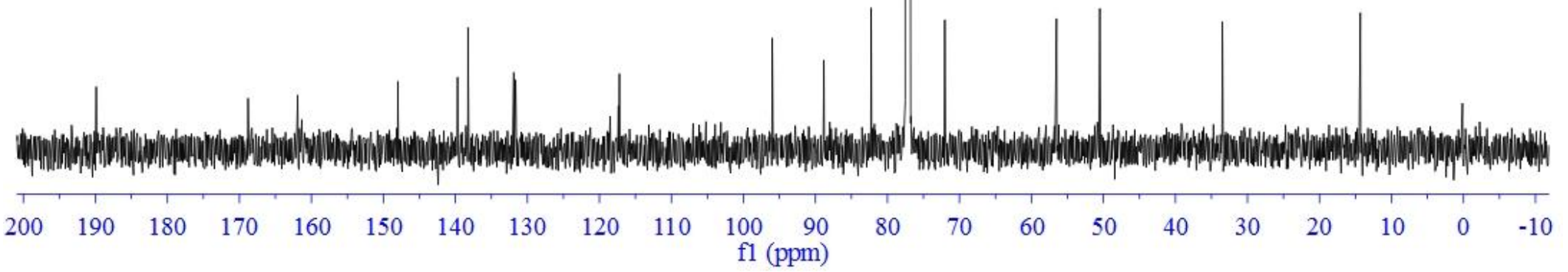


Figure S3. The HSQC Spectrum of Compound 1 in $\mathrm{CDCl}_{3}$.

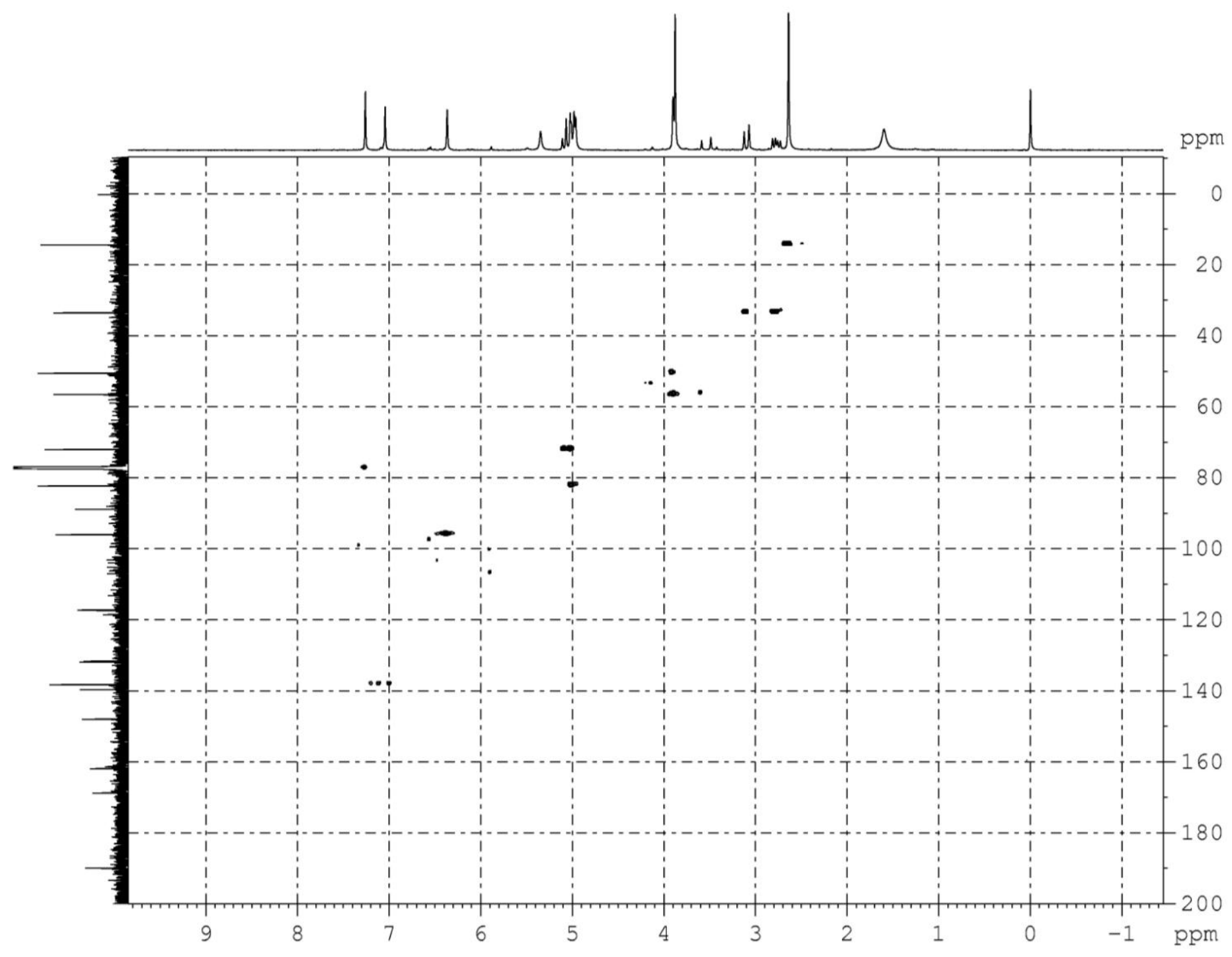


Figure S4. The HMBC Spectrum of Compound 1 in $\mathrm{CDCl}_{3}$.

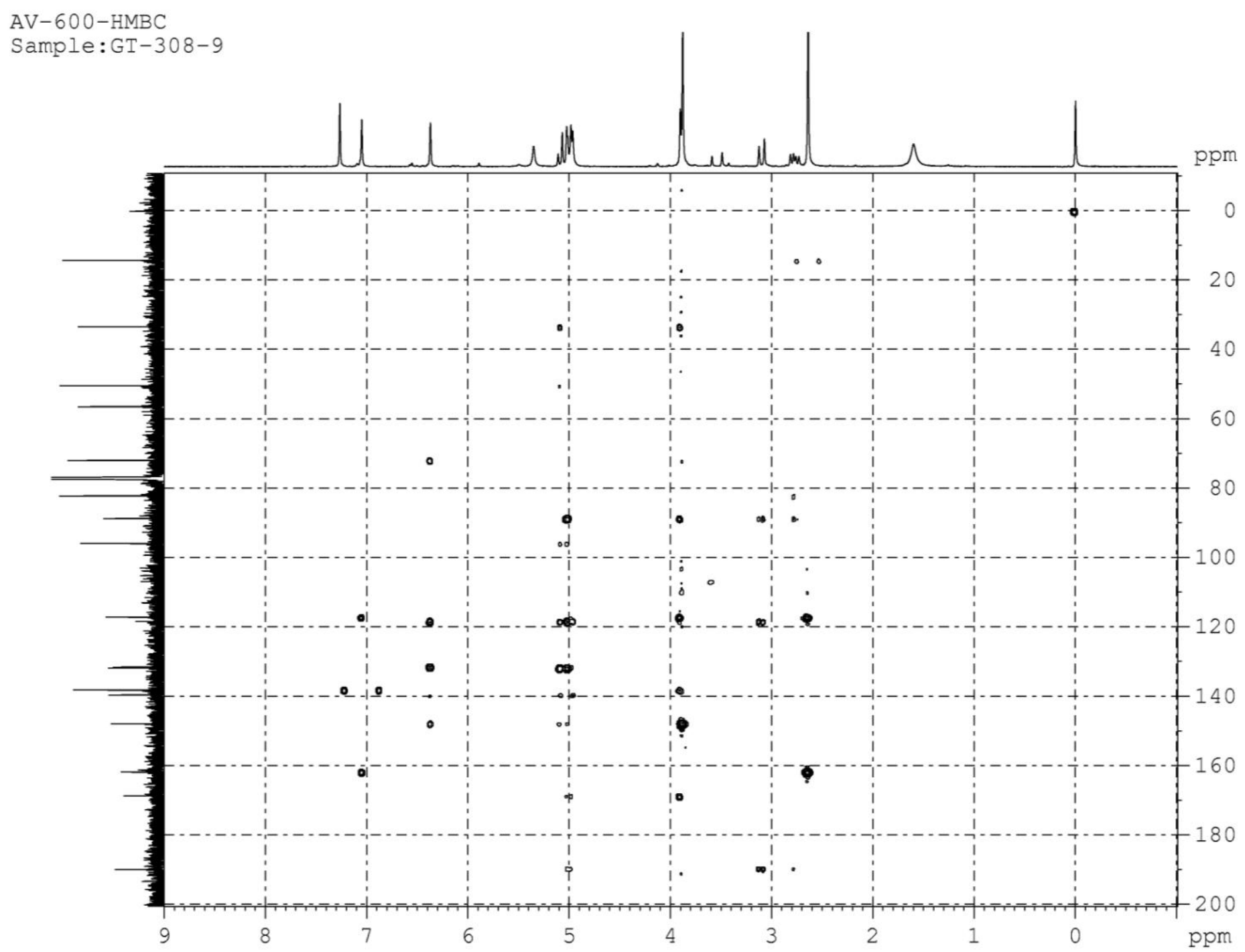


Figure S5. The ${ }^{1} \mathrm{H}-{ }^{1} \mathrm{H}$ COSY Spectrum of Compound $\mathbf{1}$ in $\mathrm{CDCl}_{3}$.

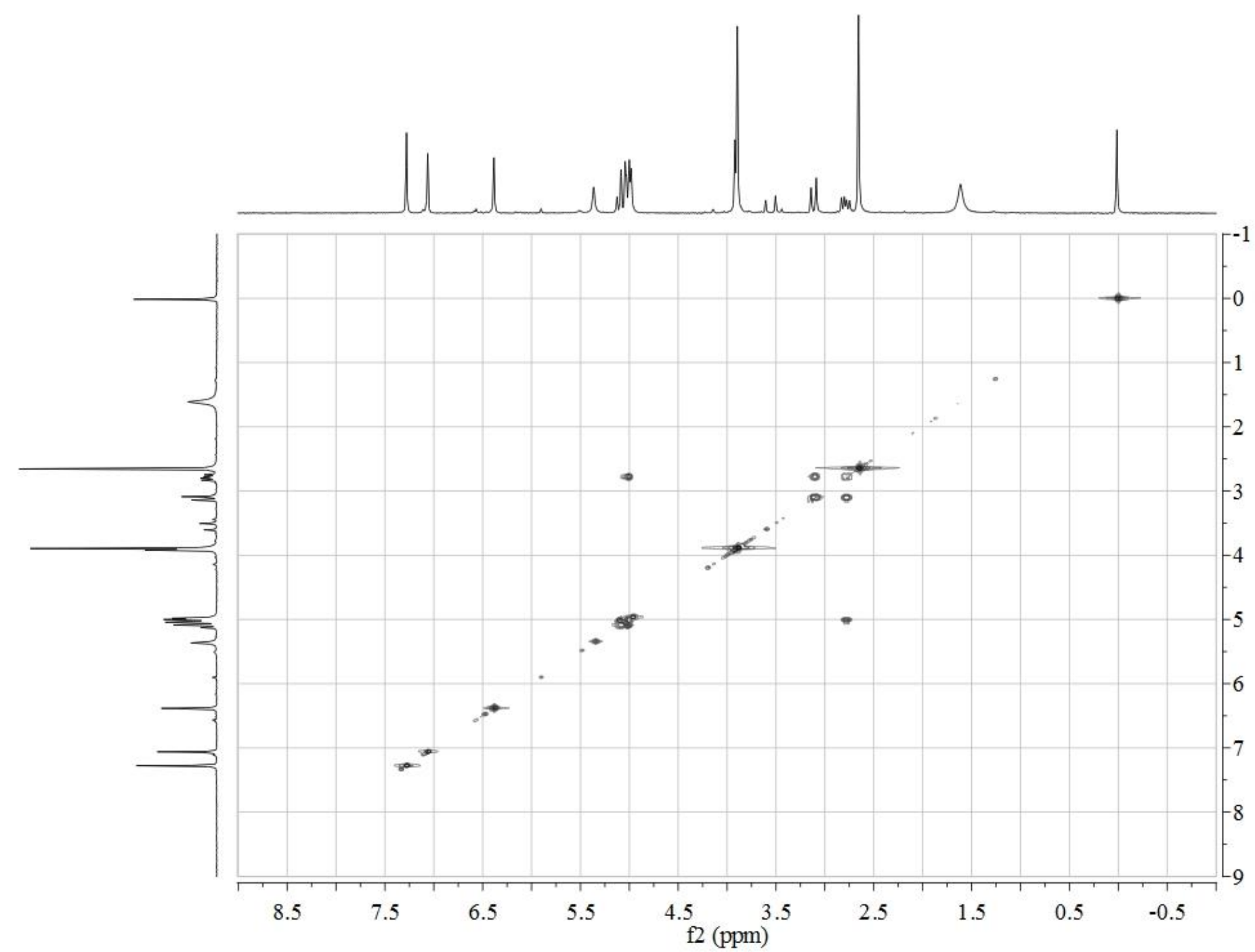


Figure S6. The NOESY Spectrum of Compound 1 in $\mathrm{CDCl}_{3}$.

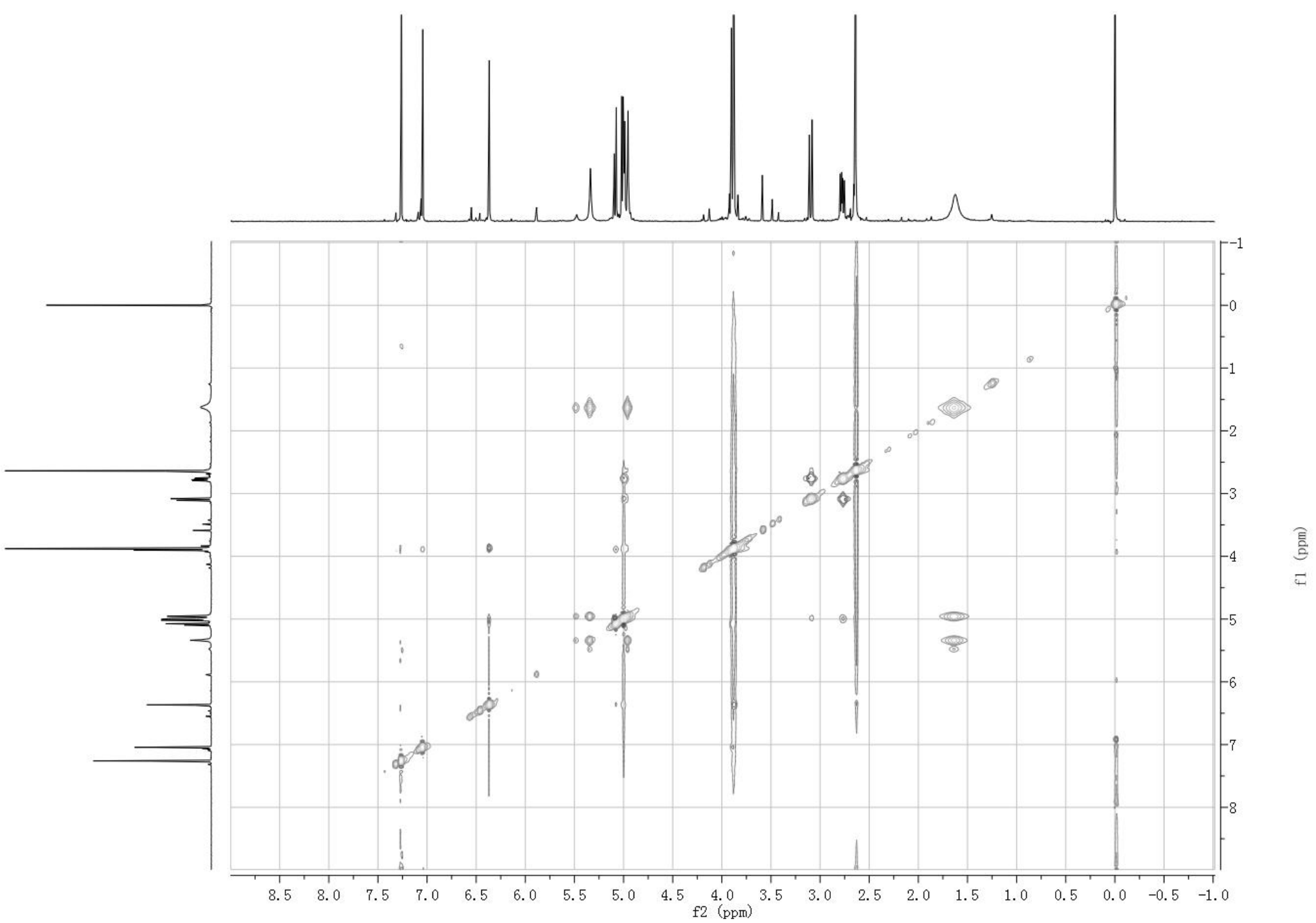


Figure S7. The ESI-MS Spectrum of Compound 1.

\section{Direct Mass Spectrometry Analysis}

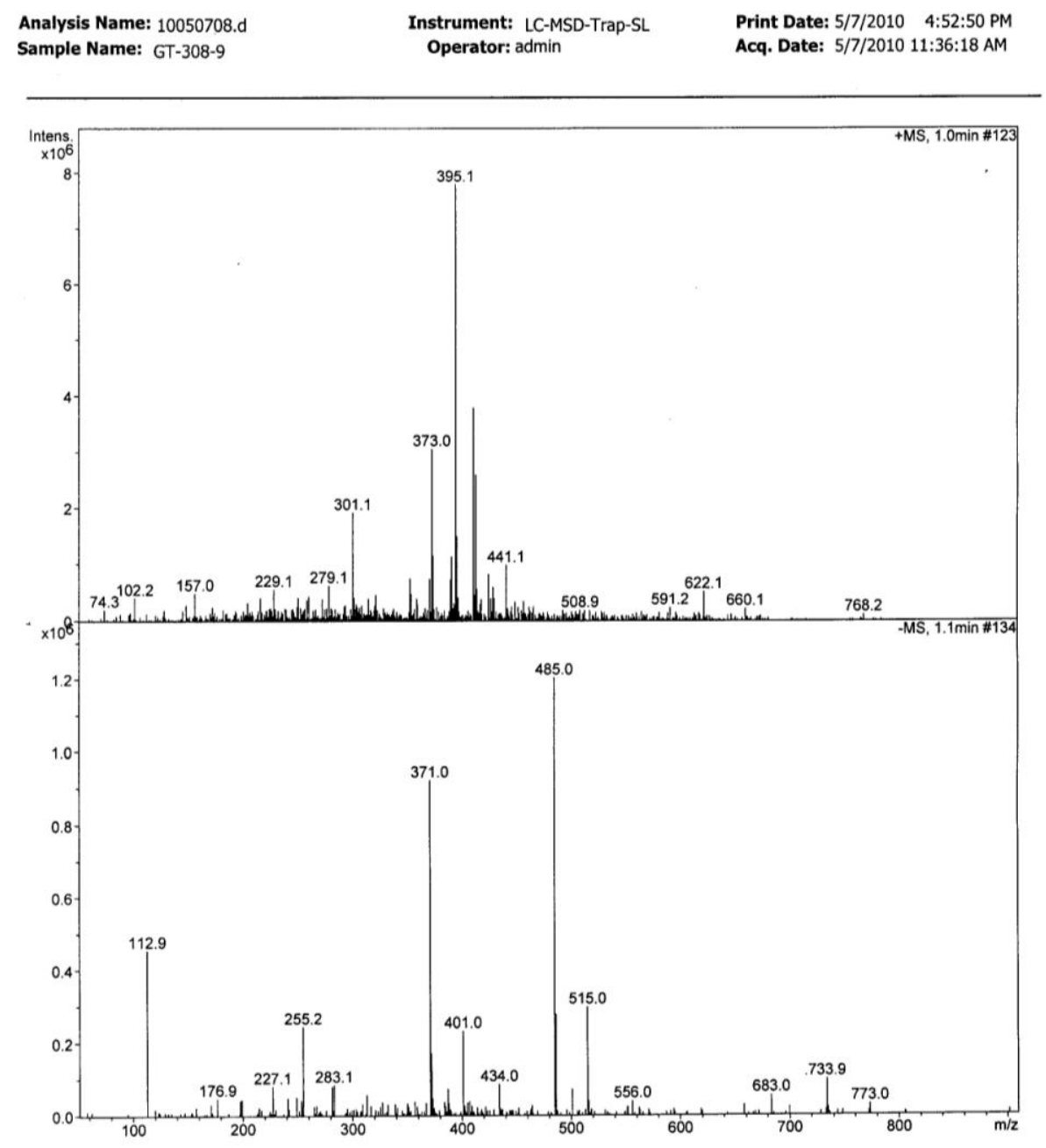


Figure S8. The HRESI-MS Spectrum of Compound 1.

\section{Elemental Composition Search Report:}

Target Mass:

Target $\mathrm{m} / \mathrm{z}=371.0767 \pm 9.00 \mathrm{ppm}$

Charge $=-1$

Possible Elements:

Element:

Exact Mass: Min: Max

C $\quad 12.000000 \quad 0 \quad 100$

N $\quad 14.003074 \quad 0 \quad 100$

$\begin{array}{llll}\text { O } & 15.994915 & 0 & 100\end{array}$

Additional Search Restrictions:

Seven Golden Rules

Nitrogen Rule

DBE Limit Mode $=$ Both Integer and Half-Integer

-Minimum DBE $=0$

-Maximum DBE $=100$

Search Results:

Number of Hits $=5$

\begin{tabular}{lccl}
$\mathrm{m} / \mathrm{z}$ & Delta $\mathrm{m} / \mathrm{z}(\mathrm{ppm})$ & $\mathrm{DBE}$ & \multicolumn{1}{l}{ Formula } \\
\hline 371.07724 & -1.46 & 12.0 & $\mathrm{C}_{19} \mathrm{H}_{15} \mathrm{O}_{8}^{-1}$ \\
371.07589 & 2.17 & 18.0 & $\mathrm{C}_{16} \mathrm{H}_{7} \mathrm{~N}_{10} \mathrm{O}_{2}^{-1}$ \\
371.07858 & -5.07 & 17.0 & $\mathrm{C}_{20} \mathrm{H}_{11} \mathrm{~N}_{4} \mathrm{O}_{4}^{-1}$ \\
371.07456 & 5.77 & 13.0 & $\mathrm{C}_{15} \mathrm{H}_{11} \mathrm{~N}_{6} \mathrm{O}_{6}{ }^{-1}$ \\
371.07992 & -8.67 & 22.0 & $\mathrm{C}_{21} \mathrm{H}_{7} \mathrm{~N}_{8}^{-1}$
\end{tabular}


Varian QFT-ESI

Mode: Negative

$\begin{array}{ll}\text { Date: } & \text { 05-AUG-2010 } \\ \text { Time: } & 15: 24: 26\end{array}$

File: GT-308-9 ESI.trans

Scans: 1

Scale: 22.8650

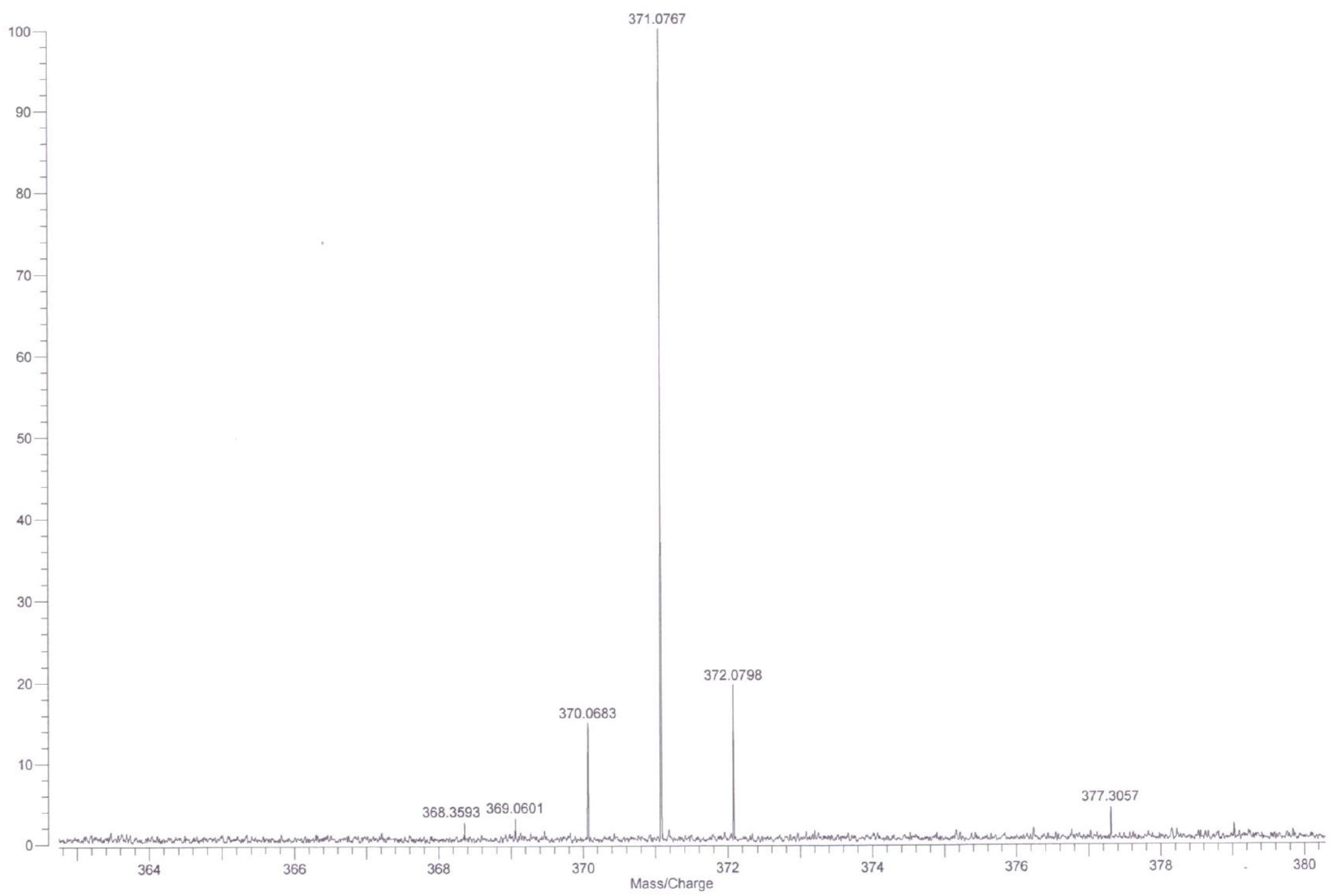




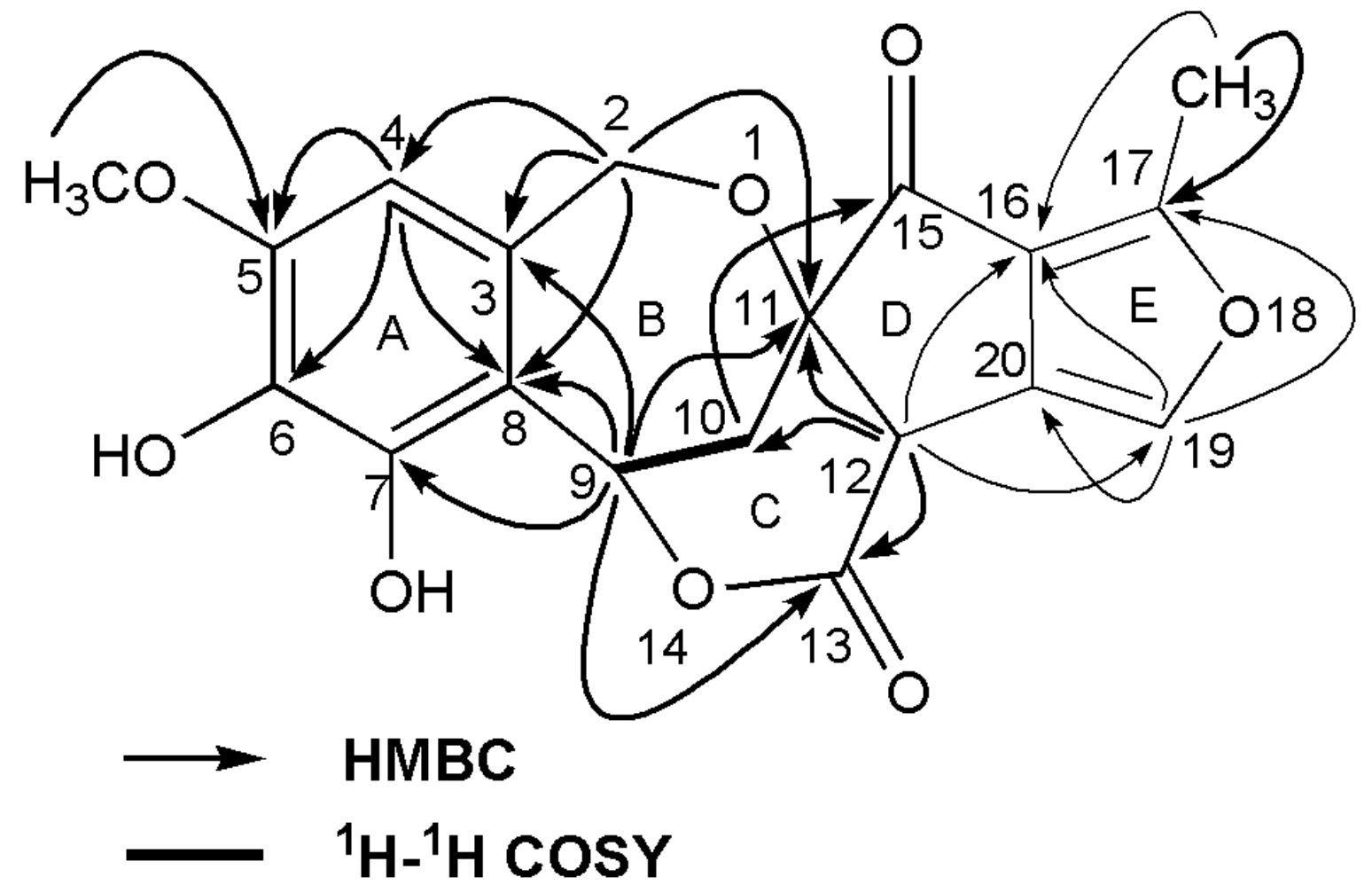


Figure S10. Key NOESY correlations of compound $\mathbf{1 .}$

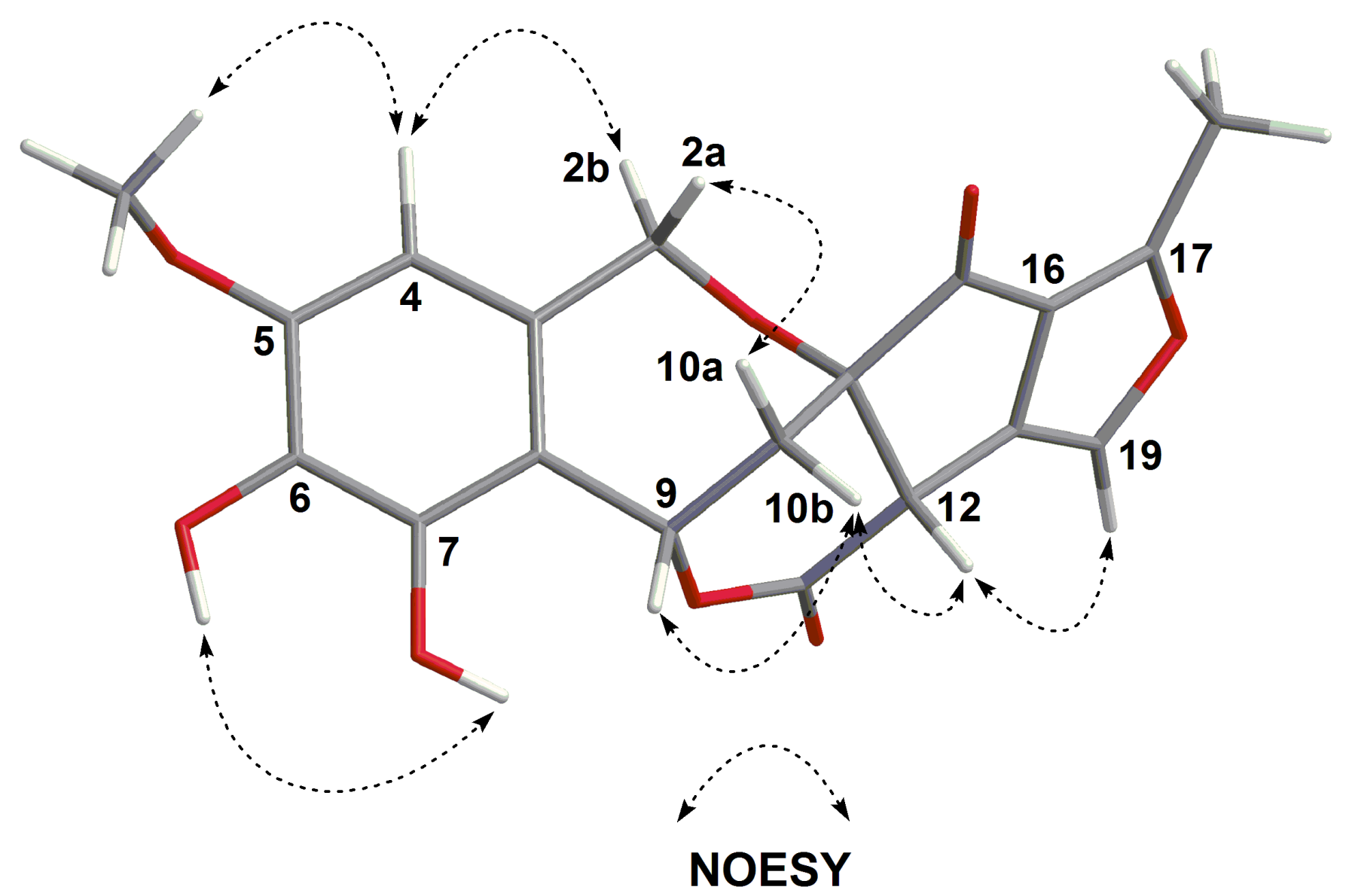


Table S1. NMR spectroscopic data of compound 1 (in $\mathrm{CDCl}_{3}$ )

\begin{tabular}{|c|c|c|c|c|}
\hline position & $\delta_{\mathrm{H}}$ mult. $(J \mathrm{~Hz})$ & $\boldsymbol{\delta}_{\mathrm{C}}$ & HMBC & NOESY \\
\hline \multirow[t]{2}{*}{2} & $\begin{array}{c}5.01(1 \mathrm{H}, \mathrm{d}, J=12.3 \mathrm{~Hz} \\
\mathrm{H}-2 \mathrm{a})\end{array}$ & $72.0(\mathrm{t})$ & C-3, C-4, C-5, C-8, C-11 & $\mathrm{H}-10 \mathrm{a}$ \\
\hline & $\begin{array}{c}5.08(1 \mathrm{H}, \mathrm{d}, J=12.3 \mathrm{~Hz} \\
\mathrm{H}-2 \mathrm{~b})\end{array}$ & & C-3, C-4, C-5, C-7, C-8, C-10 & $\mathrm{H}-4$ \\
\hline 3 & & $131.9(\mathrm{~s})$ & & \\
\hline 4 & $6.37(1 \mathrm{H}, \mathrm{s})$ & $96.0(d)$ & C-2, C-5, C-6, C-8 & $\begin{array}{c}\mathrm{H}-2 \mathrm{~b} \\
5-\mathrm{OCH}_{3}\end{array}$ \\
\hline 5 & & $148.0(\mathrm{~s})$ & & \\
\hline 6 & & $131.6(\mathrm{~s})$ & & \\
\hline 7 & & $139.7(\mathrm{~s})$ & & \\
\hline 8 & & $118.5(\mathrm{~s})$ & & \\
\hline 9 & $4.99(1 \mathrm{H}, \mathrm{d}, J=9.3 \mathrm{~Hz})$ & $82.3(\mathrm{~d})$ & $\begin{array}{c}\text { C-3, C-7, C-8, C-11, C-13, } \\
\text { C-15 }\end{array}$ & $\mathrm{H}-10 \mathrm{~b}$ \\
\hline \multirow[t]{2}{*}{10} & $\begin{array}{c}3.10(1 \mathrm{H}, \mathrm{d}, J=15.9 \mathrm{~Hz} \\
\mathrm{H}-10 \mathrm{a})\end{array}$ & $33.5(\mathrm{t})$ & C-8, C-11, C-15 & $\mathrm{H}-2 \mathrm{a}$, \\
\hline & $\begin{array}{c}2.77(1 \mathrm{H}, \mathrm{dd}, J=15.9,9.3 \\
\mathrm{Hz}, \mathrm{H}-10 \mathrm{~b})\end{array}$ & & C-9, C-11, C-15 & $\mathrm{H}-9, \mathrm{H}-12$ \\
\hline 11 & & $88.8(\mathrm{~s})$ & & \\
\hline 12 & $3.90(1 \mathrm{H}, \mathrm{m})$ & $50.5(\mathrm{~d})$ & C-10, C-11, C-13, C-16, C-19 & H-10b, H-19 \\
\hline 13 & & $168.8(\mathrm{~s})$ & & \\
\hline 15 & & $189.9(\mathrm{~s})$ & & \\
\hline 16 & & $117.2(\mathrm{~s})$ & & \\
\hline 17 & & $161.9(\mathrm{~s})$ & & \\
\hline 19 & $7.04(1 \mathrm{H}, \mathrm{s})$ & $138.3(d)$ & $\mathrm{C}-16, \mathrm{C}-17, \mathrm{C}-20$ & $\mathrm{H}-12$ \\
\hline 20 & & $117.4(\mathrm{~s})$ & & \\
\hline $5-\mathrm{OCH}_{3}$ & $3.88(3 \mathrm{H}, \mathrm{s})$ & $56.5(q)$ & C-5 & $\mathrm{H}-4$ \\
\hline $6-\mathrm{OH}$ & $4.96(1 \mathrm{H}, \mathrm{s})^{\mathrm{a}}$ & & & 7-OH \\
\hline $7-\mathrm{OH}$ & $5.35(1 \mathrm{H}, \mathrm{s})^{\mathrm{b}}$ & & & $6-\mathrm{OH}$ \\
\hline $17-\mathrm{CH}_{3}$ & $2.64(3 \mathrm{H}, \mathrm{s})$ & $14.3(\mathrm{q})$ & C-16, C-17 & \\
\hline
\end{tabular}

Note: ${ }^{\mathrm{a}, \mathrm{b}}$ These chemical shifts are interchangeable. 